University of Massachusetts Amherst

ScholarWorks@UMass Amherst

Astronomy Department Faculty Publication Series

Astronomy

2001

\title{
X-Ray Detection of Presupernova Evolution for the SN 1993J Progenitor
}

S Immler

B Aschenbach

QD Wang

University of Massachusetts - Amherst

Follow this and additional works at: https://scholarworks.umass.edu/astro_faculty_pubs

Part of the Astrophysics and Astronomy Commons

\section{Recommended Citation}

Immler, S; Aschenbach, B; and Wang, QD, "X-Ray Detection of Presupernova Evolution for the SN 1993J Progenitor" (2001). The Astrophysical Journal Letters. 1069.

$10.1086 / 324407$

This Article is brought to you for free and open access by the Astronomy at ScholarWorks@UMass Amherst. It has been accepted for inclusion in Astronomy Department Faculty Publication Series by an authorized administrator of ScholarWorks@UMass Amherst. For more information, please contact scholarworks@library.umass.edu. 
DRAFt VERSION FEBRUARY 1, 2008

Preprint typeset using LATEX style emulateapj v. 14/09/00

\title{
X-RAY DETECTION OF A PRE-SUPERNOVA EVOLUTION FOR THE SN 1993J PROGENITOR
}

\author{
Stefan Immler ${ }^{1}$, Bernd Aschenbach ${ }^{2}$ \& Q. Daniel Wang ${ }^{1}$ \\ ${ }^{1}$ Astronomy Department, University of Massachusetts, Amherst, MA 01003 \\ ${ }^{2}$ Max-Planck-Institut für extraterrestrische Physik, Postfach 1312, 85741 Garching, Germany \\ Draft version February 1, 2008
}

\begin{abstract}
We report on the first detection of a pre-supernova (SN) evolution in the X-ray regime. The results are based on ROSAT observations of SN 1993J ranging from six days to five years after the outburst. The X-ray observations are used to probe the SN shell interaction with the ambient circumstellar matter (CSM). After exploring various scenarios that might explain the observed X-ray lightcurve with a $t^{-0.27}$ rate of decline, we present a coherent picture in terms of the interaction of the SN shock front with the CSM deposited by the progenitor's stellar wind. During the observed period, the SN shell has reached a radius of $3 \times 10^{17} \mathrm{~cm}$ from the site of the explosion, corresponding to $\sim 10^{4}$ years in the progenitors stellar wind history. Our analysis shows that the mass-loss rate of the progenitor has decreased constantly from $\dot{M}=4 \times 10^{-4}$ to $4 \times 10^{-5} \mathrm{M}_{\odot} \mathrm{yr}^{-1}\left(v_{\mathrm{w}} / 10 \mathrm{~km} \mathrm{~s}^{-1}\right)$ during the late stage of the evolution. Assuming a spherically symmetric expansion, the circumstellar matter density profile is found to be significantly flatter $\left(\rho_{\mathrm{csm}} \propto r^{-1.63}\right)$ than expected for a constant mass-loss rate and constant wind velocity profile $\left(r^{-2}\right)$. The observed evolution either reflects a decrease in the mass-loss rate, an increase in the wind speed or a combination of both, indicating that the progenitor likely was making a transition from the red to the blue supergiant phase during the late stage of its evolution.
\end{abstract}

Subject headings: supernovae: individual (SN 1993J) — stars: mass loss — X-rays: individual (SN 1993J) — X-rays: ISM

\section{INTRODUCTION}

The interaction of a supernova (SN) with circumstellar medium (CSM) produces a fast shock wave in the CSM and a reverse shock wave into the outer supernova ejecta. Two characteristic regions of X-ray emission are produced by the interaction: the forward shock wave in the CSM at $T \sim 10^{4} \mathrm{~km} \mathrm{~s}^{-1}$ produces gas with $\sim 10^{9} \mathrm{~K}$, and the reverse shock wave in the supernova ejecta $\sim 10^{3} \mathrm{~km} \mathrm{~s}^{-1}$ less than the forward shock produces gas with $T \sim 10^{7} \mathrm{~K}$. The reverse shock is formed where the freely expanding supernova ejecta catches up with the CSM shocked by the blast wave. At early times, the reverse shock front is radiative and a dense, cool $\left(T<10^{4} \mathrm{~K}\right)$ shell can build up downstream from the radiating region (Chevalier \& Fransson 1994). The dense shell can absorb X-rays from the reverse shock region and reprocess them to lower energies.

This scenario was supported by observations of Type IIb SN 1993J with ROSAT (Zimmermann et al. 1994, 1996) and with ASCA (Kohmura et al. 1994). The early Xray spectrum at day 6 was hard, with $T \sim 10^{8.5} \mathrm{~K}$. At day $\sim 200$, a softer component with $T \sim 10^{7} \mathrm{~K}$ dominated. The emergence of the softer spectrum could be attributed to the decreased absorption by a cool shell (Fransson, Lundqvist \& Chevalier 1996; hereafter: FLC96).

\section{THEORETICAL BACKGROUND}

The thermal X-ray luminosity $L_{\mathrm{x}}$ of the shock heated plasma is expressed by the product of the emission measure, EM, and the cooling function, $\Lambda(T, Z, \Delta E)$, where $T$ is the plasma temperature, $Z$ represents the elemental abundance distribution, and $\Delta E$ is the X-ray energy bandwidth. For spherically symmetric conditions $\mathrm{EM}=4 \pi \int_{R_{0}}^{R} n_{\mathrm{e}}^{2} r^{2} d r$, where $r$, the radial coordinate, runs from $R_{0}$ to $R$, the current outer boundary of the shocked matter which has an electron density $n_{\mathrm{e}}$. Assuming a constant supernova shock wave speed $v_{\mathrm{s}}, R=v_{\mathrm{s}} t$, where $t$ represents the time elapsed since the explosion. If the ambient matter density $\rho$ is dominated by a wind blown by the progenitor star of the supernova the continuity equation requires $\dot{M}=4 \pi r^{2} \rho v_{\mathrm{w}}$, with $\dot{M}$ the mass loss rate and $v_{\mathrm{w}}$ the wind speed. The X-ray luminosity can then be expressed as $L_{\mathrm{x}}=\frac{1}{4 \pi} \Lambda(T) \times\left(\dot{M} / v_{\mathrm{w}}\right)^{2} \times\left(v_{\mathrm{s}} t\right)^{-1}$. We can hence use the X-ray luminosity to measure the ratio $\dot{M} / v_{\text {w }}$ of the progenitor star.

Each X-ray measurement at $t$ is related to the corresponding distance from the site of the explosion. This site had been reached by the wind at a time depending on $v_{\mathrm{w}}$, or the age of the wind $t_{\mathrm{w}}=t v_{\mathrm{s}} / v_{\mathrm{w}}$. Usually, $v_{\mathrm{s}} \gg v_{\mathrm{w}}$ so that with $t$ only a few years we can look back in time quite an appreciably large time span in the evolution of the progenitor's wind. Assuming that $v_{\mathrm{w}}$ did not change over $t_{\mathrm{w}}$ we can even directly measure the mass loss rate back in time. Whether $v_{\mathrm{w}}=$ const is a reasonable assumption will be discussed below. Integration of the mass-loss rate along the path of the expanding shell gives the mean density inside a sphere of radius $r$. For a constant wind velocity $v_{\mathrm{w}}$ and mass-loss rate $\dot{M}$, a $\rho_{\text {csm }}=\rho_{0}\left(r / r_{0}\right)^{-s}$ profile with $s=2$ is expected.

After the expanding shell has become optically thin, it is expected that emission from the SN ejecta itself, heated by the reverse shock, dominates the X-ray output of the interaction regions due to its higher emission measure and higher density. For a uniformly expanding ejecta the density structure is a function of its expansion velocity, $v$, and the time after the explosion, $t: \rho_{\mathrm{sn}}=\rho_{0}\left(t / t_{0}\right)^{-3}\left(v / v_{0}\right)^{-n}$ with $\rho_{0}$ the ejecta density at time $t_{0}$ and velocity $v_{0}$ 


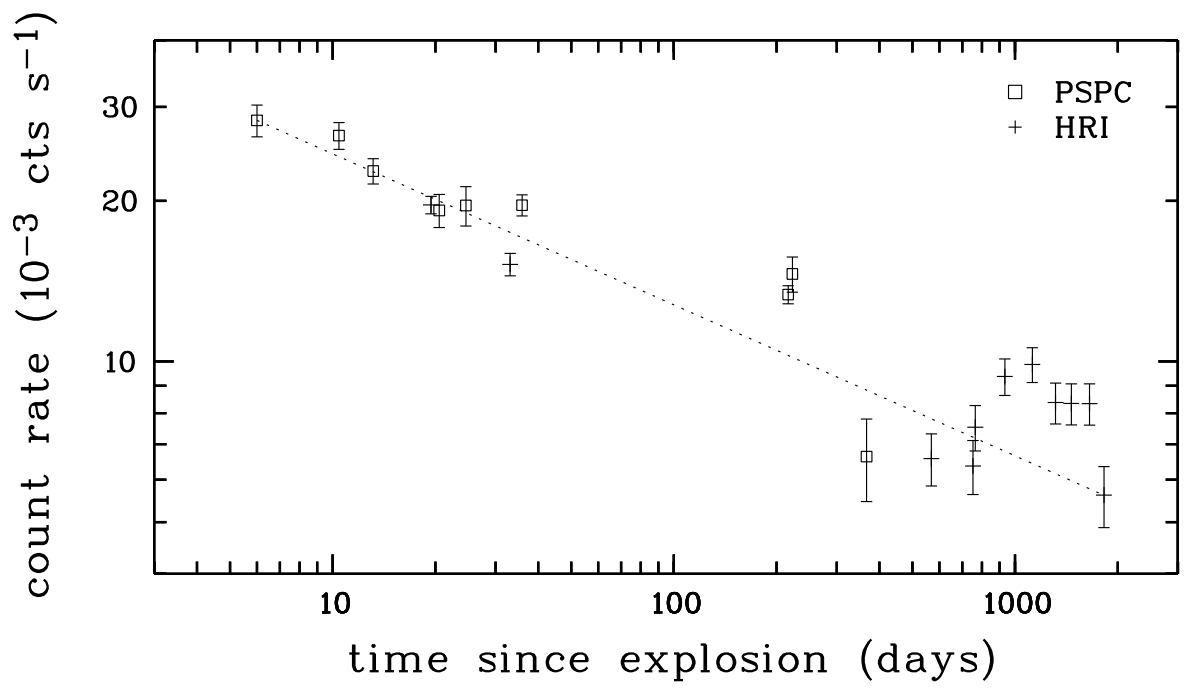

Fig. 1.- ROSAT (0.5-2 keV band) X-ray lightcurve of SN 1993J. Boxes mark PSPC data, HRI data are indicated by crosses. Error bars are $1 \sigma$ statistical errors. The dotted line illustrates a $t^{-0.27}$ rate of decline.

(FLC96). For a red supergiant progenitor, the power-law is rather steep with index $n \sim 20$ (Shigeyama et al. 1994; Baron, Hauschildt \& Branch 1994; Suzuki \& Nomoto 1995; FLC96). For constant $n$, the radius of the discontinuity surface between the forward and the reverse shock evolves in time $t$ with $R_{\mathrm{c}} \propto t^{m}$ where $m=(n-3) /(n-s)$ is the deceleration parameter.

\section{X-RAY OBSERVATIONS AND ANALYSIS}

SN 1993J in M81 was observed in 5 individual pointings with the Position Sensitive Proportional Counter (PSPC) and in 10 individual pointings with the High Resolution Imager (HRI) onboard ROSAT (Trümper 1983). Total integration times with the PSPC and HRI instruments are $52.2 \mathrm{ks}$ and $140.3 \mathrm{ks}$, respectively. Assuming an initial explosion on March 28, 1993 (Ripero 1993), the observations cover a period of 6 to 1821 days after the outburst. A detailed description of the data calibration and analysis can be found in Immler \& Wang (2001).

In order to investigate the rate of decline for SN 1993J, we constructed a combined PSPC+HRI lightcurve, which is presented in Fig. 1. HRI data were binned into 11 continuous observation blocks with 5-20 ks exposure time each to obtain satisfactory counting statistics. PSPC data were binned into 9 intervals with $2-18$ ks integration time each. The time-dependent background was determined by normalizing the total background map of the HRI and PSPC images according to the total source-removed count rate in each exposure interval. Background subtracted source counts were extracted within the $90 \%$ radii around the fixed position of SN 1993J. In order to reduce background due to UV emission and cosmic rays, only HRI PI channels 2-10 were used.

An effective $0.5-2 \mathrm{keV}$ band cooling function of $\Lambda=$ $3 \times 10^{-23}$ ergs $\mathrm{cm}^{-3} \mathrm{~s}^{-1}$ is adopted for an assumed optically thin thermal plasma with a temperature of $10^{7} \mathrm{~K}$ (Raymond, Cox \& Smith 1976). The equivalent count rate to (unabsorbed) flux conversion factor is $4 \times 10^{-11}\left(\mathrm{ergs} \mathrm{cm}^{-2} \mathrm{~s}^{-1}\right) /\left(\right.$ counts s$\left.^{-1}\right)$ for a Galacti column density of $N_{\mathrm{H}}=4 \times 10^{20} \mathrm{~cm}^{-2}$ (Dickey \& Lockman 1990). ROSAT HRI and PSPC count rates were converted using a factor of 3 appropriate for the assumed source spectrum (cf. Immler \& Wang 2001). HRI count rate to flux conversion factors as a function of spectral model parameters are given in Fig. 5 in Wang, Immler \& Pietsch (1999). The uncertainty of the HRI (PSPC) conversion factors for optically thin thermal spectra with temperature in the range $10^{7}-10^{8.5} \mathrm{~K}$ (cf. $\S 1$ ) is $22 \%(19 \%)$. An increase in absorbing column from $10^{20}$ to $10^{21} \mathrm{~cm}^{-2}$ increases the unabsorbed source fluxes by $\sim 15 \%$. Assuming a $0.86 \mathrm{keV}$ thermal Bremsstrahlung spectrum (corresponding to a temperature of $10^{7} \mathrm{~K}$ ) instead of a Raymond \& Smith thermal plasma reduces the HRI (PSPC) conversion factor by $4 \%$ (1\%).

\section{DISCUSSION}

\subsection{X-ray emission from the shock-heated stellar wind}

We will first discuss the observed X-ray lightcurve in the context of the shock-heated stellar wind model and explore other scenarios later. In order to derive the stellar wind age prior to the outburst, an initial wind velocity of $v_{\mathrm{w}}=10 \mathrm{~km} \mathrm{~s}^{-1}$ and shock front velocity of $v_{\mathrm{s}}=19900 \mathrm{~km} \mathrm{~s}^{-1}$ are assumed (Bartel et al. 1994). The choice for the stellar wind speed is justified by the fact that SN 1993J is a Type IIb SN with a massive $\left(\sim 15 M_{\odot}\right.$ ZAMS) red supergiant progenitor (e.g. Podsiadlowski et al. 1993). Typical outflow velocities for these progenitors are in the range of $3 \lesssim v_{\mathrm{w}} \lesssim 30 \mathrm{~km} \mathrm{~s}^{-1}$, peaking at $\sim 10$ $15 \mathrm{~km} \mathrm{~s}^{-1}$ (e.g. Barnbaum, Kastner \& Zuckermann 1991).

Since the shock front catches up with the wind deposited by the progenitor with a speed of up to $\sim 2000 \times$ larger, the interaction front can be used to probe the stellar wind history over a period of $\sim 10^{4}$ years. Our X-ray observations can hence be used as a 'time machine' to directly measure the CSM density during the last stages of the stellar evolution.

Fig. 2 illustrates the change in mass-loss rate as a function of the stellar wind age. It can be seen that the mass-loss rate constantly declined from $\dot{M}=4 \times 10^{-4}$ to $4 \times 10^{-5} \mathrm{M}_{\odot} \mathrm{yr}^{-1}\left(v_{\mathrm{w}} / 10 \mathrm{~km} \mathrm{~s}^{-1}\right)$ just prior to the explosion. Integration of the mass lost by the progenitor along the shock front gives the CSM density at the 
given interaction radius from the SN site. The CSM density distribution is illustrated in Fig. 3. It is found that the CSM density profile is best described by a power law $\rho_{\text {csm }} \propto r^{-s}$ with index $s=1.63 \pm 0.02$, assuming a spherically symmetric expansion. This is significantly flatter than expected for a constant mass-loss rate and constant wind velocity profile $\left(r^{-2}\right)$. The results confirm previous evidence for a rather flat CSM density profile during the first year $(s \sim 1.5$, van Dyk et al. 1994; $1.5 \lesssim s \lesssim 1.7$, FLC96; $s=1.66_{-0.25}^{+0.12}$, Marcaide et al. 1997).

The observed change in the pre-SN history could be due to variations in one of the following parameters:

- Variations in velocity and/or temperature of the shocked CSM may have caused changes in the X-ray output of the shocked region. This model was proposed to explain the radio lightcurve of SN 1993J (Fransson \& Björnsson 1998). In fact, evidence for a $\lesssim 20 \%$ decrease in the expansion velocity over the first $\sim 4$ years was reported (Marcaide et al. 1997; Bartel et al. 2000). This change in shock expansion velocity, however, would only lead to a $<10 \%$ increase in the mass-loss rate and cannot account for the observed order-of-magnitude change. Also, as the ROSAT soft X-ray band cooling function for optically thin thermal plasma emission is not very sensitive to the plasma temperature $\left(\Lambda(T) \propto T^{0.5}\right.$; e.g. Raymond, Cox \& Smith 1976), a change in temperature from $10^{7} \mathrm{~K}$ to a few $\times 10^{11} \mathrm{~K}$ would be required to explain the observed change in the mass-loss rate history. Such drastic temperature variations have neither been observed for $\mathrm{SNe}$ nor have they been put forward by models describing the CSM interaction. Therefore, variations in either the shock expansion velocity or in the temperature accounting for the inferred evolution can be ruled out.

- A different scenario would be a non-spherically symmetric geometry caused by a binary evolution of the progenitor. Observational evidence that the progenitor of SN 1993J was a stripped supergiant in a binary system has been presented based on the visual lightcurve, which cannot be described by a single, massive star alone (e.g. Podsiadlowski et al. 1993). In addition, "double-horned" emission line profiles indicate the presence of a flattened or disk-like expanding shell (Matheson et al. 2000a; 2000b). The X-ray lightcurve also shows some significant deviations from the long-term $t^{-0.27}$ behavior (cf. Fig. 1). Such deviations have also been observed for SN 1979C in the radio regime (Montes et al. 2000) and were discussed in the context of a self-colliding binary stellar wind model leading to the formation of a multiple shell-like CSM profile (Schwarz \& Pringle 1996). X-ray observations of SN 1979C, however, were not suited to address these questions (Immler, Pietsch \& Aschenbach 1998; Kaaret 2001). Similarly, a binary model for SN 1993J cannot be challenged by the ROSAT data alone and no information about the long-term radio lightcurve of SN 1993J is available.

- Alternatively, the observed evolution is caused by changes in the wind parameters. The X-ray lightcurve implies $\rho_{\text {csm }} \propto r^{-s}$ with $s=1.63$, or, using the continuity equation, $\dot{M} / v_{\mathrm{w}} \propto r^{2-s} \propto r^{0.37} \propto t_{\mathrm{w}}^{0.37}$. The ratio $\dot{M} / v_{\mathrm{w}}$ therefore decreases with time approaching the date of the explosion, indicating either a decrease in the mass-loss rate, an increase in the wind speed or a combination of both. It is interesting to compare typi- cal wind parameters for red and blue supergiants, which are on the same evolutionary track for massive $\left(\gtrsim 15 M_{\odot}\right.$ ZAMS) stars (e.g. Salasnich, Bressan \& Chioso 1999). Whereas the mass-loss rate at $t_{\mathrm{w}} \sim 10^{4}$ years $(\dot{M}=$ $\left.4 \times 10^{-4} \mathrm{M}_{\odot} \mathrm{yr}^{-1}\left(v_{\mathrm{w}} / 10 \mathrm{~km} \mathrm{~s}^{-1}\right)\right)$ is typical for a red supergiant (e.g. $\dot{M}=3 \times 10^{-4} \mathrm{M}_{\odot} \mathrm{yr}^{-1}$ for HD 179821, Jura, Velusamy \& Werner 2001; Chin \& Stothers 1990; Schaller et al. 1992), the observed change in ratio $\dot{M} / v_{\mathrm{w}}$ by an order of magnitude at $t_{\mathrm{w}} \sim 30$ years could indicate a transition between the red and blue supergiant phase due to an increase in effective temperature of the star. Blue supergiants are known to have significantly lower mass-loss rates and higher wind velocities $\left(\dot{M} \sim 10^{-6} \mathrm{M}_{\odot} \mathrm{yr}^{-1}\right.$, $v_{\mathrm{w}} \sim 500-1000 \mathrm{~km} \mathrm{~s}^{-1}$; e.g. Kudritzki et al. 1999). This scenario for the evolution of the SN 1993J progenitor would have some interesting similarities with that of SN 1987A, whose progenitor completely entered the blue supergiant phase after significant mass-transfer to a companion (Podsiadlowski et al. 1993).

Whereas our X-ray data alone cannot give information on whether $\dot{M}$ or $v_{\mathrm{w}}$ have effectively changed during the late stage of the progenitor's evolution, there is a clear difference between these possibilities regarding the kinetical energy of the stellar wind: using $\rho_{\mathrm{csm}} \propto r^{-1.63}$, the kinetical wind energy decreased by a factor of 13 for a changing $\dot{M}$ during the observed $\Delta t_{\mathrm{w}} \sim 10^{4}$ years. In the scenario of a changing $v_{\mathrm{w}}$ only, an increase in kinetical wind energy by a factor of $10^{2.2}$ is expected. Future stellar evolutionary models might give answers as to which of the two possibilities is more likely.

\subsection{X-ray emission from the shocked-heated SN ejecta}

Let us explore the scenario that the X-ray emission is dominated by the ejecta, heated by the reverse shock. A necessary condition for the ejecta accounting for the observed X-ray emission is that the absorption by the postshock gas is low. In this model, an ejecta density structure of $\rho_{\mathrm{sn}}=\rho_{0}\left(t / t_{0}\right)^{-3}\left(v / v_{0}\right)^{-n}$ is expected (FLC96). The total X-ray output of both the reverse-shock heated ejecta and the shocked stellar wind in the forward shock is then given by $L_{\mathrm{x}} \propto\left(\dot{M} / v_{\mathrm{w}}\right)^{2} \times\left(t / t_{0}\right)^{3-2 s}$ in the case where $n$ is large. In fact, using our inferred $\mathrm{X}$-ray rate of decline of $L_{\mathrm{x}} \propto t^{-0.27}$ is entirely consistent with the above $L_{\mathrm{x}} \propto t^{3-2 s} \propto t^{-0.24}$ for $s=1.63$. The assumption that $n$ must be large is hence confirmed by our data and has also been concluded from radio and optical observations of SN 1993J ( $n \sim 20-30$; Shigeyama et al. 1994; Baron, Hauschildt \& Branch 1994; Suzuki \& Nomoto 1995; FLC96). It is important to note that if the density gradient for the ejecta is large, X-rays from the reverse shock must be heavily absorbed. Whereas variations in temperature of the ejecta can be ruled out to explain the observed X-ray lightcurve (cf. $\$ 4.1$ ), a change in absorption from initially $10^{22} \mathrm{~cm}^{-2}$ to the Galactic column of $4 \times 10^{20} \mathrm{~cm}^{-2}$ could account for the observed decrease in source flux (cf. Fig. 5 in Wang, Immler \& Pietsch 1999). The signature of high absorption, however, is absent in all ROSAT PSPC spectra of SN 1993J, which are consistent with the Galactic foreground absorption $\left(N_{\mathrm{H}}=5.3 \pm 1.7 \times 10^{20} \mathrm{~cm}^{-2}\right.$, Zimmermann et al. 1994, 1996). The most likely scenario is hence that the emission from the reverse shock is com- 
pletely absorbed and that the observed soft X-rays are only due to the shocked CSM of the progenitor wind.

Based on the modeling of the early X-ray lightcurve of SN 1993J, Fransson, Lundqvist \& Chevalier (FLC96) concluded that the X-ray emission during the first months originates from the interaction of the SN shock with the circumstellar medium. As the reverse shock begins to penetrate the cool shell it is expected to contribute an increasing fraction to the total X-ray output. This predicted rise of the ROSAT band X-ray flux after $\sim 100$ days (as illustrated in Figs. 8 and 10 in FLC96) due to the emerging ejecta is not observed with ROSAT. Instead, the overall long-term X-ray lightcurve (Fig. 1) is declining with a power-law close to the initial measurements, which were consistently explained as a result of the interaction of the SN shell with the ambient CSM.

\section{CONCLUSIONS}

We present the first detection of a pre-SN evolution in X-ray, based on long-term monitoring of SN 1993J with ROSAT over a period of 5 years. The data are fully consistent with a description in the context of the SN shock interacting with the CSM blown off by the progenitor's stellar wind. From the X-ray rate of decline $L_{\mathrm{x}} \propto t^{-0.27}$ we infer a CSM profile $\rho_{\text {csm }} \propto r^{-1.63}$, which is significantly flatter than expected for a constant mass-loss rate and constant wind velocity profile $\left(r^{-2}\right)$. The observations cover an epoch of $\sim 10^{4}$ years in the progenitor's stellar wind history. During this period, the mass-loss rate of the progenitor has decreased constantly from $\dot{M}=4 \times 10^{-4}$ to $4 \times 10^{-5} \mathrm{M}_{\odot} \mathrm{yr}^{-1}\left(v_{\mathrm{w}} / 10 \mathrm{~km} \mathrm{~s}^{-1}\right)$ just prior to the explosion. The most likely explanation for this pre-SN evolution is either an increase in wind speed, a decrease in mass-loss rate or a combination of both, indicative that the progenitor star was undergoing a transition from the red to the blue supergiant phase. The data demonstrate the scientific potential of long-term X-ray monitoring of SNe as an important diagnostical tool to probe the CSM interaction and the evolution of SN progenitors.

This research made use of various online services and databases, e.g. ADS, HEASARC, NED, and the ROSAT data archive at MPE. The project is supported by NASA under the grants NAG 5-8999 and NAG5-9429.

\section{REFERENCES}

Barnbaum, C., Kastner, J.H., \& Zuckermann, B. 1991, AJ, 102, 289

Baron, E., Hauschildt, P.H., \& Branch, D. 1994, ApJ, 426, 334

Bartel, N., et al. 1994, Nature, 368, 610

Bartel, N., et al. 2000, Science, 287, 112

Chevalier, R.A., \& Fransson, C. 1990, ApJ, 420, 268

Chin, C.-W., \& Stothers, R.B. 1994, ApJS, 73, 821

Dickey, J.M., \& Lockman, F.J. 1990, ARA\&A, 28, 215

van Dyk, S.D., Weiler, K., Sramek, R.A., Rupen, M.P., \& Panagia, N. 1994, ApJ, 432, L115

Fransson, C., Lundqvist, P., \& Chevalier, R.A. 1996, ApJ, 461, 993 (FLC96)

Fransson, C., \& Björnsson, C.-I. 1998, ApJ, 509, 861

Immler, S., Pietsch, W., \& Aschenbach, B. 1998, A\&A, 331, 601

Immler, S., \& Wang, Q.D. 2001, ApJ, 554, 202

Jura, M., Velusamy, T., \& Werner, M.W. 2001, ApJ 556, 408

Kaaret, P. 2001, ApJ accepted, astro-ph/0106568

Kohmura, Y., et al. 1994, PASJ, 46, L157

Kudritzki, R.P., et al. 1999, A\&A, 350, 970

Marcaide, J.M., et al. 1997, ApJ, 486, L31
Matheson, T., et al. 2000a, ApJ, 120, 1487

Matheson, T., et al. 2000b, ApJ, 120, 1499

Montes, M.J., et al. 2000, ApJ, 532, 1124

Podsiadlowski, Ph., Hsu, J.J.L., Joss, P.C., \& Ross, R.R. 1992, Nature, 364, 509

Raymond, J.C., Cox, D.P., \& Smith, B.W. 1976, ApJ, 204, 290

Ripero, J. 1993, IAU Circ., No. 5731

Salasnich, B., Bressan, A., \& Chioso, C. 1999, A\&A, 342, 131

Schaller, G., Schaerer, D., Meynet, G., \& Maeder, A. 1992, A\&AS, 96, 269

Schwarz, D.H., \& Pringle, J.E. 1996, MNRAS, 282, 1018

Shigeyama, T., Suzuki, T., Kumagai, S., et al. 1994, ApJ, 420, 341

Suzuki, T., \& Nomoto, K. 1995, ApJ, 455, 658

Trümper, J. 1983, Adv. Space Res., 2, 241

Wang, Q.D., Immler, S., \& Pietsch, W. 1999, ApJ, 523, 121

Zimmermann, H.U., et al. 1994, Nature, 367, 621

Zimmermann, H.U., Lewin, W.H.G., \& Aschenbach, B. 1996, in Conf Proc. "Röntgenstrahlung from the Universe", MPE Report 263, 298 


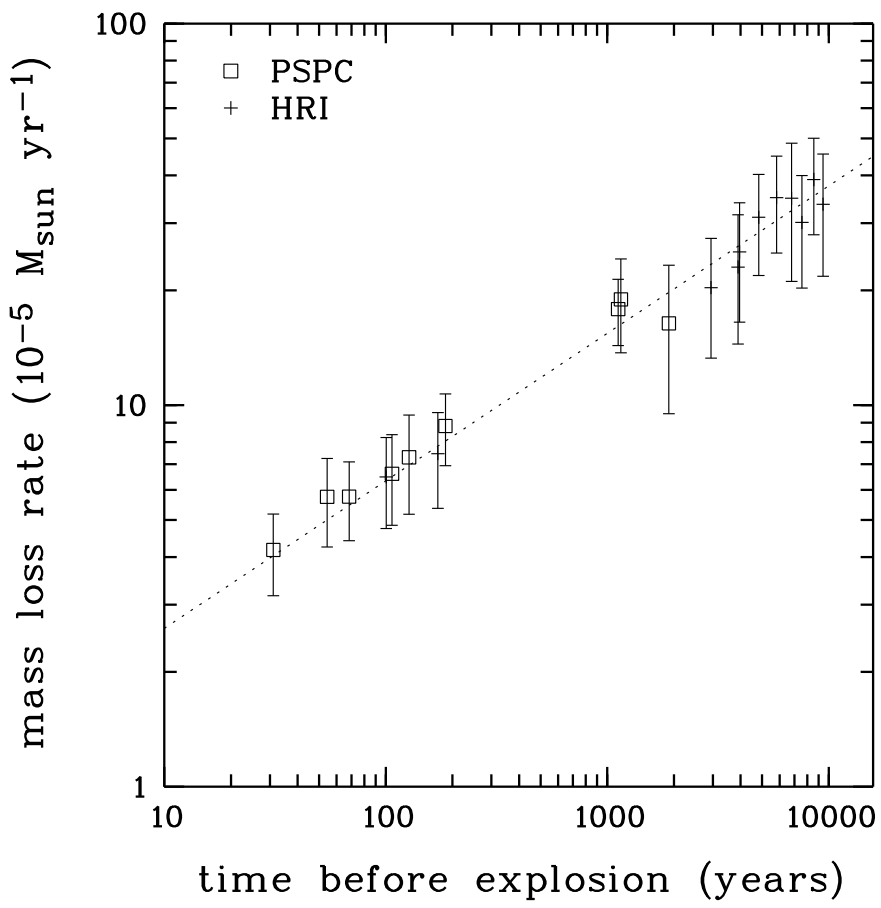

FIG. 2.- Mass-loss rate history of the SN 1993J progenitor. PSPC data are marked by boxes, HRI data are indicated by crosses.

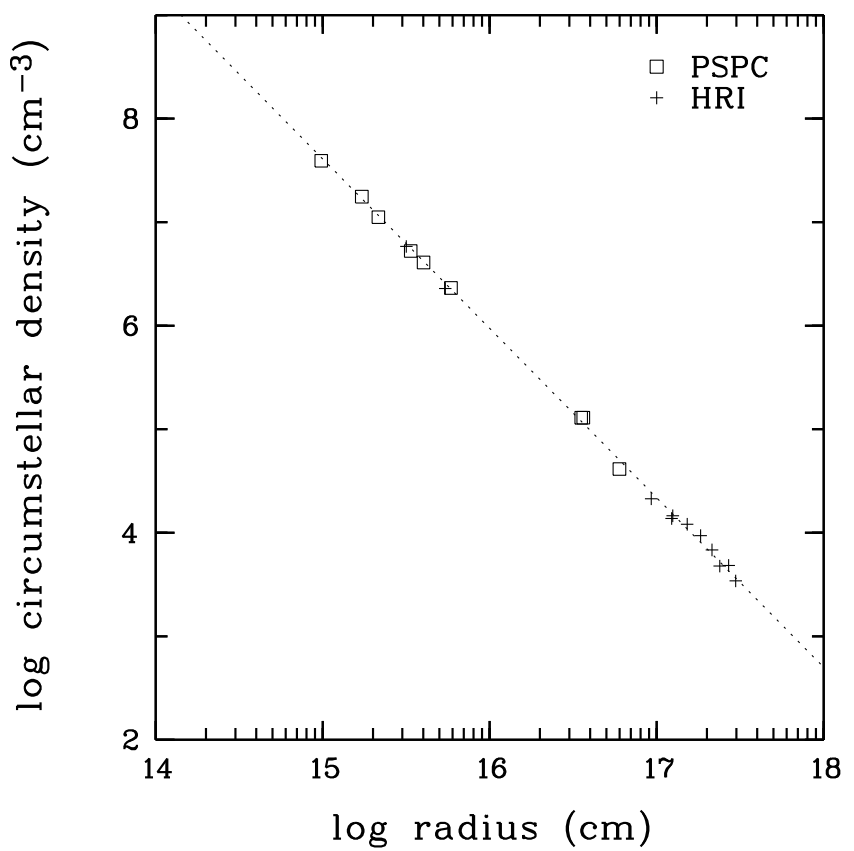

FIG. 3.- Circumstellar density profile as a function of SN shell expansion radius. The solid line gives the best-fit CSM profile of $\rho_{\text {csm }} \propto r^{-1.63}$ to the PSPC (boxes) and HRI (crosses) data points. 